This item was submitted to Loughborough's Research Repository by the author.

Items in Figshare are protected by copyright, with all rights reserved, unless otherwise indicated.

\title{
The measurement of approximate number system acuity across the lifespan is compromised by congruency effects
}

\section{PLEASE CITE THE PUBLISHED VERSION}

https://doi.org/10.1177/1747021818779020

\section{PUBLISHER}

SAGE Publications (C) Experimental Psychology Society

\section{VERSION}

AM (Accepted Manuscript)

\section{PUBLISHER STATEMENT}

This work is made available according to the conditions of the Creative Commons Attribution-NonCommercialNoDerivatives 4.0 International (CC BY-NC-ND 4.0) licence. Full details of this licence are available at: https://creativecommons.org/licenses/by-nc-nd/4.0/

\section{LICENCE}

CC BY-NC-ND 4.0

\section{REPOSITORY RECORD}

Norris, Jade E., Sarah Clayton, Camilla K. Gilmore, Matthew Inglis, and Julie Castronovo. 2019. "The Measurement of Approximate Number System Acuity Across the Lifespan Is Compromised by Congruency Effects". figshare. https://hdl.handle.net/2134/32944. 


\section{The Measurement of Approximate Number System Acuity across the Lifespan is Compromised by Congruency Effects}

Jade Eloise Norris ${ }^{1 *}$, Sarah Clayton ${ }^{2}$, Camilla Gilmore ${ }^{3}$, Matthew Inglis ${ }^{3}$, Julie Castronovo ${ }^{4^{*}}$

\footnotetext{
${ }^{1}$ Department of Psychology, University of Bath, Claverton Down, Bath, BA2 7AY, UK

${ }^{2}$ Department of Health Sciences, University of Leicester, University Road, Leicester, LE1 7RH, UK

${ }^{3}$ Mathematics Education Centre, Loughborough University, Loughborough, Leicestershire, LE11 3TU, UK

${ }^{4}$ School of Life Sciences, University of Hull, Cottingham Road, Hull, HU6 7RX, UK
}

*Correspondence: Jade Eloise Norris, Department of Psychology, University of Bath, Claverton Down, Bath, BA2 7AY, UK. j.norris@bath.ac.uk | 01225384841

- The data were collected at the Department of Psychology, University of Hull

Word count: 5,278

Keywords: Approximate Number System, Number Sense, Ageing, Convex Hull, Visual Cues, Numerosity Discrimination 


\section{$1 \quad$ Abstract}

2 Recent studies have highlighted the influence of visual cues such as dot size and cumulative surface 3 area on the measurement of the approximate number system (ANS). Previous studies assessing ANS 4 acuity in ageing have all applied stimuli generated by the Panamath protocol, which does not control nor measure the influence of convex hull. Crucially, convex hull has recently been identified as an

6 influential visual cue present in dot arrays, with its impact on older adults' ANS acuity yet to be 7 investigated. The current study therefore investigated the manipulation of convex hull by the 8 Panamath protocol, and its effect on the measurement of ANS acuity in younger and older 9 participants. Firstly, analyses of the stimuli generated by Panamath revealed a confound between 10 numerosity ratio and convex hull ratio. Secondly, although older adults were somewhat less 11 accurate than younger adults on convex hull incongruent trials, ANS acuity was broadly similar 12 between the groups. These findings have implications for the valid measurement of ANS acuity 13 across all ages, and suggest that the Panamath protocol produces stimuli that do not adequately control for the influence of convex hull on numerosity discrimination. 
The Approximate Number System (ANS) supports the imprecise representation of numerosity, as demonstrated by behavioural and neuronal indicators of Weber's law: i) numerical representations become less precise and more approximate with increasing magnitude (the size effect), and ii) discrimination between two numerosities becomes more difficult as their ratio approaches 1 (the ratio effect) (Dehaene, 1997; Gallistel \& Gelman, 2000; Izard, Sann, Spelke, \& Streri, 2009; Piazza \& Izard, 2009; Piazza, Izard, Pinel, Le Bihan, \& Dehaene, 2004). The acuity of the ANS is most often measured with comparison tasks, whereby participants are shown two arrays of non-symbolic numerosities (e.g. dots), and asked to select which array is most numerous without counting. Comparing performance on easier and harder ratios provides evidence for the ratio effect. However, the validity of such tasks in providing a pure measure of ANS acuity is contested, because participants are found to be influenced by visual characteristics of the stimuli including convex hull (the perimeter around a dot set, sometimes referred to as "area extended"), average dot size, and cumulative surface area of the dots (Clayton \& Gilmore, 2014; Gebuis \& Reynvoet, 2012a, 2012b, 2012c; Gilmore, Cragg, Hogan, \& Inglis, 2016; Leibovich \& Henik, 2013; Szúcs, Nobes, Devine, Gabriel, \& Gebuis, 2013). It is generally accepted that when these visual characteristics are uncontrolled, i.e. the more numerous set is also larger in terms of its non-numerical visual cues, participants may make their decisions using visual cues alone (e.g. by choosing the array that contains larger dots on average compared to the other array), without engaging the ANS (Gebuis \& Reynvoet, 2012a, 2012b). Here we explore how these visual cue characteristics are manipulated in a commonly-used programme to generate dot array stimuli, and how this impacts on numerosity judgements across the lifespan.

When creating dot array stimuli, researchers originally sought to address concerns about the influence of visual cues by applying controls to average dot size and cumulative surface area, varying the relationship of these visual cues with the number of dots in the array (Abreu-Mendoza, SotoAlba, \& Arias-Trejo, 2013). For example, as described in the software guidelines for Panamath (Halberda, Mazzocco, \& Feigenson, 2008), a commonly used method for generating stimuli for nonsymbolic numerosity comparison tasks, dot-size congruency is controlled by manipulating the cumulative surface area of the arrays. During a congruent trial, cumulative surface area is positively correlated with numerosity. The more numerous array therefore has a larger cumulative surface area and a larger average dot size: cumulative surface area and dot size are both congruent to numerosity. During what we will term a matched trial (to reduce confusion between an 'incongruent' trial as defined by Halberda et al. (2008) and incongruent visual cues in the more 
general sense), cumulative surface area is matched between arrays in order that the less numerous array has a larger average dot size: dot size is incongruent to numerosity. Finally, during anticorrelated trials, cumulative surface area (and therefore average dot size too) are negatively correlated with, and so incongruent to, numerosity (Halberda et al., 2008). In short, only dot size is incongruent to numerosity during matched trials, whereas during anticorrelated trials both cumulative surface area and average dot size are incongruent to numerosity. The application of such controls has varied between studies using a range of protocols to generate dot arrays: whilst some have included congruent and matched trials (Cappelletti, Didino, Stoianov, \& Zorzi, 2014), others have compared congruent and anticorrelated trials (Clayton, Gilmore, \& Inglis, 2015; Gilmore et al., 2013; Hurewitz, Gelman, \& Schnitzer, 2006; Inglis \& Gilmore, 2014; Odic, Libertus, Feigenson, \& Halberda, 2013; Szúcs et al., 2013), with others using all three control conditions (DeWind \& Brannon, 2012; Fuhs \& McNeil, 2013; Keller \& Libertus, 2015; Rousselle \& Noël, 2008) or matched trials alone (Gray \& Reeve, 2014). Varied methods of visual cue control, along with other inconsistencies such as display time, number of trials, and numerosity ratio cause problems when comparing ANS acuity across studies (Clayton \& Gilmore, 2014; Clayton et al., 2015; Dakin, Tibber, Greenwood, Kingdom, \& Morgan, 2011; Dietrich, Huber, \& Nuerk, 2015; Gebuis \& Reynvoet, 2012c; Gilmore et al., 2016; Inglis \& Gilmore, 2013, 2014; Szűcs et al., 2013).

Several authors have argued that during trials with incongruent visual cues, participants must first inhibit the influence of those visual cues in order to perform a numerosity judgement (Cappelletti et al., 2014; Cappelletti, Pikkat, Upstill, Speekenbrink, \& Walsh, 2015; Clayton \& Gilmore, 2014; Fuhs \& McNeil, 2013; Gilmore et al., 2013, 2016). However, others find similar performance between congruent trials and those with incongruent visual cues, arguing that performance on ANS tasks does not require inhibitory control (Keller \& Libertus, 2015; Odic, Hock, \& Halberda, 2014; Odic et al., 2013). This is important when considering ANS acuity in ageing. Inhibitory control declines with age (Hasher \& Zacks, 1988; Kramer, Humphrey, Larish, \& Logan, 1994): if incongruent visual cues in ANS tasks do indeed require inhibition, then older participants may be expected to show a greater decline in performance on such trials. A limited number of studies have investigated ANS acuity in ageing, with some examining the impact of congruency effects. Halberda, Ly, Wilmer, Naiman, and Germine (2012) investigated ANS acuity across the lifespan, concluding that acuity declines with increasing age beyond 30 years. However, it is difficult to draw conclusions from this study regarding the impact of older age for three reasons. Firstly, participants aged 45-85 were categorised within one age group, due to the small number of older adults included in the overall sample. Secondly, Figure 3 (p.11119: Halberda et al., 2012) demonstrates highly variable ANS acuity and several outliers within the older group. Thirdly, although congruent and matched trials were used, whether 
age-related decline may be attributable to poorer performance during matched trials, where inhibitory control may be required, is not reported. Indeed, in the first study to directly investigate the impact of dot-size congruency on ANS acuity in ageing, Cappelletti et al. (2014) compared younger and older adults' performances on an ANS task based on the Halberda et al. (2008) Panamath protocol. Their findings initially indicated declined ANS acuity in older age (as in Halberda et al., 2012). However, separate analyses for performances on congruent and matched trials revealed that the older group's acuity was only declined compared to the younger group when average dot size was incongruent to numerosity (matched trials). The authors concluded that seemingly poorer ANS acuity in ageing may be accounted for by declined inhibitory control (Hasher \& Zacks, 1988) rather than deteriorated approximate numerical skills. In short, older adults' ability to inhibit the influence of an incongruent visual cue (dot size) was found to be declined. A later study by Cappelletti et al. (2015) administered ANS training paired with parietal stimulation to younger groups at pre-training, with improvement in both groups after training. Crucially, older adults' stronger ANS acuity post-training was driven by improved performance for matched trials (i.e. with incongruent dot size), which was related to smaller interference effects on traditional inhibition tasks. Moreover, older adults' success in learning to inhibit non-numerical magnitudes on the ANS task led to poorer performance on tasks assessing the discrimination of such magnitudes (e.g. length discrimination). These findings further support the existence of an inhibitory component to ANS tasks, a finding which may be particularly evident in older adults due to age-related decline in inhibitory control (Cappelletti et al., 2014; Hasher \& Zacks, 1988). However, another study using the same protocol and comparable methods found similar ANS acuity for younger and older adults, even for matched trials (Norris, McGeown, Guerrini, \& Castronovo, 2015). It is likely that other methodological differences such as the use of intermixed vs separated dot displays contributed to these contradictory findings (see Norris \& Castronovo, 2016 for evidence of the impact of different stimuli presentation methods in younger adults).

Although some of the studies investigating ANS acuity in ageing have examined the impact of dot size congruency, recently the influence of convex hull congruency on numerosity discrimination has been emphasised (Clayton \& Gilmore, 2014; Clayton et al., 2015; Gebuis \& Reynvoet, 2012c; Gilmore et al., 2016). Convex hull refers to the smallest possible perimeter that can be drawn around an array (Graham, 1972), and may affect the processing of numerosity to a greater extent than dot size, even when convex hull and numerosity are not correlated (Gebuis \& Reynvoet, 2012c). As with dot size, convex hull can be congruent (the more numerous array has the larger convex hull), or incongruent to numerosity (the more numerous array has the smaller convex hull). Crucially, the 
117 studies reviewed above which investigated ANS acuity in ageing did not investigate convex hull 118 congruency effects, as they used stimuli generated by the Panamath protocol, which does not 119 control for convex hull congruency. Clayton and Gilmore (2014) investigated how manipulating numerosity mediated the influence of visual cues on 7-9 year-olds' ANS acuity. As numerosity increased, performance declined due to the increasing interference of convex hull. However for smaller numerosities, performance was most strongly influenced by dot size. Therefore, the type of visual cues used by participants appears to be mediated by numerosity (Clayton \& Gilmore, 2014). Crucially, below-chance performance during larger-numerosity trials demonstrated the greater influence of convex hull over other visual cues. In a further study, Clayton et al. (2015) compared ANS acuity when measured with two commonly-used protocols: Panamath, which controls total cumulative surface area in order to manipulate dot size (Halberda et al., 2008), and a script by Gebuis and Reynvoet (2011) which controls both the cumulative surface area and convex hull of dot arrays. The authors not only found poorly correlated performance between the protocols, but also diverging interactions between cumulative surface area and convex hull congruencies: for the Gebuis and Reynvoet (2011) paradigm, accuracy was higher for convex hull incongruent trials when cumulative surface area was congruent compared to when it was incongruent. However, cumulative surface area did not significantly affect performance when convex hull was congruent. Therefore, convex hull congruency appears to influence numerosity comparison performance to a greater extent than cumulative surface area congruency with the Gebuis and Reynvoet (2011) paradigm. Performance was also enhanced during convex hull congruent trials on the Panamath task. However on the Panamath task, accuracy was higher for cumulative surface area incongruent trials compared to cumulative surface area congruent trials regardless of convex hull. Finally, Gilmore et al. (2016) demonstrated that although dot size influences children's performance on an ANS task, this effect decreases into adulthood, whereas the influence of convex hull remains consistent from childhood to adulthood. These findings emphasise the influence of convex hull during ANS tasks, highlighting the necessity to investigate the effect of convex hull on protocols which do not control it, such as Panamath (see also DeWind \& Brannon, 2016). Indeed, as performance on the Panamath task is significantly influenced by convex hull (Clayton et al., 2015; DeWind \& Brannon, 2016), it is unclear to what extent previous findings of age-related decline in ANS acuity may be due to poorer performance on convex hull-incongruent trials.

The influence of convex hull on ANS acuity as measured by the Panamath protocol is therefore a timely and important consideration in examining the inhibitory components of ANS tasks. Moreover, as the studies to date investigating the impact of ageing on ANS acuity have all used stimuli 
151 protocol which does not manipulate nor measure the impact of convex hull, it is important to consider the influence of convex hull on the conclusions drawn in these studies: that older adults' poorer inhibitory control leads to declined performance during dot-size incongruent trials

154 (Cappelletti et al., 2014, 2015). It is therefore necessary to examine whether similar mechanisms 155 may shape the impact of convex hull congruency on older adults' performances, especially 156 considering recent findings indicating that convex hull constitutes a more important predictor of ANS 157 task performance compared to other visual cues (Clayton \& Gilmore, 2014; Clayton et al., 2015; 158 Gebuis \& Reynvoet, 2012c). Therefore, the primary aim of the current study was to further examine 159 the nature of the visual cues in the stimuli generated by the Panamath protocol, with a particular 160 focus on the way in which convex hull varies. Secondly, the study investigated the extent to which 161 convex hull congruency affects ANS task performance for older and younger adults. 
Forty participants were recruited, 20 older adults aged 62-70 (14 females; $M_{\text {age }}=65, S D=2.9$ ) and 20 younger adults aged 18-24 (16 females; $M_{\text {age }}=20, S D=1.8$ ). Younger participants were recruited through the Department of Psychology at the University of Hull and received course credit. Older participants were recruited by the first author from the local community and participated voluntarily. The study was approved by the Department of Psychology Ethics Committee at the University of Hull. All participants were fully informed of the study aims and provided written consent.

\section{Screening}

Participants were screened at recruitment for a history of psychiatric disorder, depression, or abnormal vision. Older adults were administered the Mini Mental State Exam (MMSE: Folstein, Folstein, \& McHugh, 1975) with a score $<27 / 30$ providing a cut-off for exclusion. The Geriatric Depression Scale (GDS: Yesavage et al., 1982) was administered to all participants, with a score $>5$ providing a cut-off point (as in Norris et al., 2015). No participants were excluded due to scores beyond cut-off on the MMSE or GDS.

Procedure

Approximate Number System acuity was measured using the downloadable Panamath software (Halberda et al., 2008). Two spatially separate arrays of between 5 and 21 yellow and blue dots were presented simultaneously side-by-side on a grey background for $200 \mathrm{~ms}$ (yellow on the left, blue on the right), followed by a $200 \mathrm{~ms}$ backward mask of randomly distributed yellow and blue pixels. Participants initiated each trial using the space bar, and were asked to decide which array was more numerous (yellow or blue). Participants responded as quickly as possible without sacrificing accuracy using the ' $A$ ' (yellow) and ' $L$ ' (blue) keys, which were covered with correspondingly-coloured dots. The dot stimuli were generated with two within-subject factors: visual cue control with 3 levels (congruent [both cumulative surface area and average dot size are congruent to numerosity], matched [cumulative surface area is matched and average dot size is incongruent], and anticorrelated [both cumulative surface area and average dot size are incongruent]), and numerosity ratio bin with 4 levels $(1.1-1.19,1.19-1.28,1.32-1.43$, and 2.28-2.47; ratio bins $1,2,3$, and 4 respectively). There were 420 trials in total. Convex hull size and convex hull congruency were calculated post hoc for each trial by using the Graham (1972) scan algorithm on screenshots of each 
194 trial as generated by Panamath. This calculation also summed the total number of yellow and blue 195 pixels, providing a measure of the cumulative surface area of each array. 
197 We first report the visual characteristics of the stimuli generated by the Panamath protocol, 198 followed by an examination of the impact of these characteristics on young and older adults' performance on the ANS task.

In order to control for the effect of visual cues, Panamath is designed to generate three types of stimuli: congruent (cumulative surface area and average dot size positively correlate with numerosity), matched (cumulative surface area is matched to numerosity, and average dot size negatively correlates with numerosity) and anticorrelated (cumulative surface area and dot size negatively correlate with numerosity). However, when we calculated the cumulative surface area of the arrays by summing the number of blue and yellow pixels, we discovered that the matched trials were not precisely matched in terms of cumulative surface area: the more numerous array always had a greater cumulative surface area (mean pixel number difference $=150$, range $=2-592$ ) . Therefore in matched trials, cumulative surface area was actually congruent, even though in some cases there was only a small pixel-number difference. Consequently, for our analyses we collapsed the three Panamath conditions (congruent, matched, and anticorrelated) into two (cumulative surface area congruent [congruent and matched] vs. cumulative surface area incongruent [anticorrelated]). ${ }^{1}$

With the convex hull of the arrays calculated for each trial, we sought to investigate to what extent the Panamath protocol produced equally-weighted convex hull congruent and incongruent trials, and how this was affected by other factors within the protocol (numerosity ratio and cumulative surface area).

218 Figure 1 depicts the relationships between within-subjects factors on the Panamath protocol.

219 Numerosity ratio here refers to (left set/right set), rather than (larger set/smaller set) as defined by Panamath, so that cumulative surface area ratio, convex hull ratio and numerosity ratio were calculated in the same manner. The correlation between convex hull ratio and numerosity ratio $(r=$ $.720,95 \% \mathrm{Cl}[.671, .763], p<.001)$ demonstrates a confound between within-subject factors on the Panamath protocol: convex hull ratio increases with increasing numerosity ratio.

\footnotetext{
${ }^{1}$ Analysing the data with the original three levels of congruency as defined by the Panamath protocol did not affect the direction or the significance of the results.
} 
226 Moreover, an examination of the Panamath-defined numerosity ratio bins not only replicates the

227 finding that convex hull ratio (and therefore convex hull congruency) increases alongside numerosity

228 ratio, but also indicates that all trials in ratio bin 4 had a congruent convex hull (see Table 1). Indeed,

229 convex hull was congruent for the majority of all trials (335/420).

230 Table 1: Number of trials per Panamath-defined numerosity ratio bin in the convex hull congruent 231 and incongruent conditions

\section{Convex hull}

Numerosity Ratio (Bin)

\section{Congruent}

71

1.19-1.28 (2)

$1.32-1.43(3)$

2.28-2.47 (4)

80

79

105
34

Incongruent

25

26

0

ANS acuity

234 The following analyses focus on accuracy as the dependent variable for ANS acuity, because 235 accuracy is thought to provide the most reliable and valid measure (Clayton et al., 2015; Guillaume, 236 Gevers, \& Content, 2015; Inglis \& Gilmore, 2014). As would be expected due to the ratio effect on 237 numerosity discrimination, there was a positive by-items correlation between accuracy and 238 numerosity ratio $(r=.563,95 \% \mathrm{Cl}[.494, .625], p<.001)$. Next, we investigated the effect of age 239 group, cumulative surface area congruency, and convex hull congruency on accuracy. A mixed 240 ANOVA was conducted with cumulative surface area congruency (congruent, incongruent) and 241 convex hull congruency (congruent, incongruent) as within-subjects factors, and age group (older, younger) as a between-subjects factor. There were no main effects of cumulative surface area congruency $\left(F(1,38)=3.185, p=.082, \eta_{p}^{2}=.077, \mathrm{BF}_{\text {inclusion }}=1.07^{2}\right)$ or age group $(F(1,38)=.628, p=$

\footnotetext{
${ }^{2}$ The inclusion Bayes Factor compares the evidence in support of each effect by comparing across all possible models including the effect with all possible models without the effect. This was calculated in JASP.
} 
$\left..433, \eta_{p}^{2}=.016, \mathrm{BF}_{\text {inclusion }}=0.48\right)$, and no interaction between age group and cumulative surface area congruency $\left(F(1,38)=1.891, p=.177, \eta_{p}^{2}=.047, \mathrm{BF}_{\text {inclusion }}=0.50\right)$. However, accuracy was significantly higher for convex hull congruent trials $(M=83.25 \%, S D=37.34)$ than for convex hull incongruent trials $\left(\mathrm{M}=72.09 \%, \mathrm{SD}=44.86: F(1,38)=258.190, p<.001, \eta_{p}^{2}=.872, \mathrm{BF}_{\text {inclusion }}>\right.$ 10^305). Moreover, convex hull congruency interacted with cumulative surface area congruency $\left(F(1,38)=5.005, p=.031, \eta_{p}^{2}=.116, \mathrm{BF}_{\text {inclusion }}=2.28\right.$, as in Clayton et al., 2015): Figure 2 demonstrates that when convex hull was congruent, performance between cumulative surface area congruent $(M=83.40 \%, S D=37.21)$ and incongruent trials $(M=83.10 \%, S D=37.48)$ was similar $(p=$ .604 , Cohen's $d=.012$ : LSD pairwise comparisons). However during convex hull incongruent trials, participants tended to respond more accurately when cumulative surface area was incongruent compared to when it was congruent ( $p=.038$, Cohen's $d=-.064)$.

The interaction between convex hull congruency and age group was significant $(F(1,38)=4.328, p=$ $\left..044, \eta_{p}^{2}=.102, \mathrm{BF}_{\text {inclusion }}=1.02\right)$. Although accuracy on convex hull congruent trials was similar for the younger $(M=83.22 \%, S D=37.37)$ and older groups $(M=83.29 \%, S D=37.32 ; p=.968$, Cohen's $d$ $=.002$ : LSD pairwise comparisons), younger adults outperformed older adults when convex hull was incongruent (younger: $M=73.35 \%, S D=44.22$; older: $M=70.82 \%, S D=45.47$ ), although this difference did not reach significance $(p=.200$, Cohen's $d=.056$ : LSD pairwise comparisons, see Figure 3$).^{3}$

Finally, the interaction between cumulative surface area congruency, convex hull congruency, and age group did not reach significance $\left(F(1,38)=1.610, p=.212, \eta_{p}^{2}=.041, \mathrm{BF}_{\text {inclusion }}=0.67\right)$.

\footnotetext{
${ }^{3}$ In light of the evidence for the influence of convex hull during numerosity discrimination in ageing, we reanalysed the findings from our previous study, where ANS acuity was similar for younger and older adults regardless of dot-size congruency (Norris, McGeown, Guerrini, \& Castronovo, 2015). Convex hulls were calculated using the Graham (1972) scan algorithm. Responses to convex hull incongruent trials $(M=78.00 \%, S D=41.44)$ were found to be significantly less accurate than to convex hull congruent trials $\left(M=91.16 \%, S D=28.40: F(1,48)=231.077, p<.001, \eta_{p}^{2}=.828\right)$. Moreover, there was a marginal interaction between convex hull congruency and age group $\left(F(1,48)=4.017, p=.051, \eta_{p}^{2}=.077\right)$, due to a tendency for poorer performance during convex hull incongruent trials for the older group compared to the younger group. Crucially, the impact of convex hull congruency on performance for all participants was significant, whereas the effect of dot size congruency was not.
} 
267 The current study investigated the impact of visual cue congruency on ANS acuity as measured by the Panamath protocol in a group of younger and older adults. For the first time, we investigated patterns of both cumulative surface area congruency and convex hull congruency on trials generated by the Panamath protocol and their impact on older adults' ANS acuity. Although convex hull congruency has been found to affect numerosity processing to a greater extent than the visual cues that have been more frequently controlled in previous research (e.g. cumulative surface area and dot size) (Clayton \& Gilmore, 2014; Clayton et al., 2015; Gebuis \& Reynvoet, 2012c; Gilmore et al., 2016), to date, only the impact of dot-size congruency on ANS acuity in ageing has been examined. In some studies, poorer inhibitory control has been proposed to account for declined performance during dot size-incongruent trials in older age (Cappelletti et al., 2014, 2015). These studies had used stimuli generated by the Panamath protocol, which does not control convex hull congruency. The current study therefore explored the visual characteristics of stimuli generated by the Panamath protocol and their impact on the measurement of ANS acuity, whilst directly investigating whether older adults performed more poorly when convex hull was incongruent. The current findings demonstrate that the Panamath protocol produces dot arrays that are confounded between convex hull ratio and numerosity ratio. There was some evidence that older adults appeared to be more susceptible to the influence of convex hull information when making numerosity judgements, but the key test of this effect was only borderline significant $(p=.044)$. Potential explanations for these findings are discussed below.

Our analyses indicate that the Panamath protocol generates stimuli which favour a congruent over an incongruent convex hull, an effect which becomes more pronounced as numerosity ratio increases. These findings have clear implications for studies using the Panamath protocol. Because convex hull is congruent on most trials, this may improve overall performance on the task. Crucially, the current findings demonstrate that numerosity ratio and convex hull ratio can be confounded on stimuli generated by the Panamath protocol. This affects the valid and reliable measurement of ANS acuity because participants could perform at above-chance levels on dot comparison tasks purely by responding on the basis of convex hull information and without the need to engage in numerosity processing. Our findings also support the suggestion that participants integrate multiple visual cues during numerosity discrimination (Clayton et al., 2015; Gebuis \& Reynvoet, 2012b), resulting in interactions when visual cues vary in their congruency with numerosity: when convex hull was incongruent to numerosity, participants were more accurate when cumulative surface area was also 
incongruent (as in Clayton et al., 2015; Gebuis \& van der Smagt, 2011; Keller \& Libertus, 2015). The

300 findings therefore emphasise the necessity of considering the impact of non-numerical visual cues 301 and the interactions between such cues during numerosity comparison.

302 ANS acuity

303 Reflecting previous findings, overall ANS acuity was similar between age groups, with no age-related 304 decline in performance on cumulative surface area incongruent trials (as in Norris et al., 2015). 305 Performance was poorer for all participants during convex hull incongruent trials. Although the convex hull congruency effect appeared to be more pronounced for the older group compared to the younger group, this effect was small and was not well-supported by the Bayesian analysis compared to the overall influence of convex hull for all participants. Previous findings of stronger dot-size congruency effects for older compared to younger adults may suggest that similar findings should emerge for convex hull congruency. One possible explanation for the weak evidence in the current study of a stronger convex hull congruency effect in older age may be that, due to the confounded nature of the Panamath protocol, a relatively small number of convex hull incongruent trials were generated. In previous studies, larger proportions of dot-size incongruent trials have been used. Therefore, stronger evidence for the interaction between convex hull congruency and age group, and indeed even a group difference for overall ANS acuity may have emerged had the number of convex hull congruent and incongruent trials been equally-weighted. It is well established from studies of inhibition tasks that the overall proportion of congruent and incongruent trials impacts on the size of congruency effects (Logan \& Zbrodoff, 1979). Crucially here, these effects are not consistent in younger and older adults. West and Baylis (1998) found that the difference between younger and older adults on a Stroop task was greater when the task consisted mostly of incongruent trials compared with mostly congruent trials. It is possible therefore that the small proportion of incongruent trials in the task used here may have masked differences between younger and older adults that could be apparent in a more evenly-balanced version of the task.

324 Overall however, our results support the suggestion that convex hull affects numerosity 325 discrimination to a greater extent than dot-size or cumulative surface area when measuring ANS 326 acuity (Clayton \& Gilmore, 2014; Clayton et al., 2015; Gebuis \& Reynvoet, 2012c; Gilmore et al., 327 2016).

\section{Methodological and Theoretical Conclusions}

329 The current study highlights that significant confounds may exist in the dot array stimuli produced by 330 the Panamath protocol, indicating that trials are overall more likely to be convex hull congruent, 
possibly facilitating performance. A confound between convex hull ratio and numerosity ratio raises concerns about the validity and reliability of non-symbolic numerosity comparison tasks conducted with stimuli generated by the Panamath protocol. Moreover, in light of recent claims that the Panamath protocol does not produce congruency effects (Keller \& Libertus, 2015; Odic et al., 2014, 2013), our investigation indicates that researchers may have been focusing on the wrong visual cue: convex hull appears to affect numerosity processing over and above dot-size and cumulative surface area. Controlling only dot size is therefore insufficent (as in the Panamath protocol and other stimuli generation methods used in the literature: e.g. Dehaene, Izard, \& Piazza, 2005), as multiple visual cues may be simultaneously extracted from dot arrays during numerosity discrimination (Clayton et al., 2015; Gebuis \& Reynvoet, 2012a, 2012c). Here we found that convex hull appears to exert more of an influence on ANS task performance compared to the other visual cues present in a numerosity display. Previous investigations, using different methods of generating dot stimuli, have found that several visual cues (total circumference, convex hull, density, and cumulative surface area) influence numerosity judgements over and above the influence of numerosity information (Leibovich \& Henik, 2014).

The current findings also have implications for studies reporting a link between ANS acuity and mathematical achievement. As researchers have used a range of visual cue-controls and numerosity ratios in generating dot arrays, it is unclear to what extent relationships with mathematical achievement may in fact reflect a link with the inhibitory control required to ignore convex hull (Clayton \& Gilmore, 2014; Clayton et al., 2015; Fuhs \& McNeil, 2013; Gilmore et al., 2013, 2016; Norris \& Castronovo, 2016; Szúcs et al., 2013). Indeed, as studies assessing the ANS in children often use easier (i.e. larger) numerosity ratios, convex hull may facilitate performance to an even greater extent in these studies (as convex hull is more likely to be congruent). The current study therefore highlights the necessity for researchers to seriously consider the influence of convex hull on numerosity discrimination when exploring its relationship with maths achievement (Clayton et al., 2015; Gilmore et al., 2016). Moreover, the results raise questions regarding previous conclusions of declined ANS acuity in ageing (Halberda et al., 2012), and whether dot-size congruency can fully account for these effects (Cappelletti et al., 2014; Norris et al., 2015). Future research must investigate whether ANS acuity is declined in ageing when convex hull is systematically controlled: should older adults' performances on more stringently-controlled paradigms (e.g. Gebuis \& Reynvoet, 2011) be declined compared to younger participants, this would support the suggestion that ANS tasks involve inhibitory control (Clayton \& Gilmore, 2014; Fuhs \& McNeil, 2013; Gilmore et 
extent than dot size and cumulative surface area (Clayton \& Gilmore, 2014; Clayton et al., 2015; Gebuis \& Reynvoet, 2012c; Gilmore et al., 2016).

Finally, the current findings contribute to the theoretical debate surrounding the extent to which numerosity is the cue primarily extracted from dot arrays over and above the other approximate quantities present (the non-numerical visual cues). On one hand, some argue that numerosity is the primary cue extracted from non-symbolic arrays, and that numerosity therefore drives performance on dot discrimination tasks, as opposed to the other non-symbolic quantities present in the array (e.g. convex hull, dot size, cumulative surface area: Barth et al., 2006; Halberda et al., 2008). On the other hand, the competing processes account (Gilmore et al., 2013) proposes that non-numerical visual cues are extracted during numerosity discrimination, and that participants must inhibit their influence in order to then respond to numerosity. It is therefore proposed that numerosity and the other visual cues present in non-symbolic arrays must compete to be processed, with two possible outcomes: firstly, participants may respond based on the salience of various visual cues (bigger dots, larger convex hull). Secondly, if participants are able to inhibit a response to these salient visual cues, then they can respond to numerosity. In the current study, convex hull congruency affected numerosity discrimination performance: these findings therefore provide further evidence that participants must inhibit convex hull when it is incongruent before being able to respond to numerosity (Clayton \& Gilmore, 2015; Gilmore et al., 2013), supporting the competing processes hypothesis. In addition, some researchers have suggested that numerosity isn't primarily extracted during ANS tasks, but rather a weighted combination of non-numerical visual cues is used by participants to discriminate between dot arrays (Gebuis \& Gevers, 2011; Gebuis \& Reynvoet, 2012b, 2012c). The current study cannot rule out this suggestion. Although our data indicate a numerosity ratio effect, in considering the confounded nature of the stimuli, this doesn't necessarily indicate that numerosity is the primary cue being extracted. Because the majority of trials were convex hull congruent, participants may not need to extract numerosity to make a correct discrimination on the majority of trials. Indeed, participants could most often discriminate between the arrays using convex hull and achieve above-chance overall accuracy for the current study. Our findings therefore further highlight the need to directly investigate the influence of convex hull on numerosity discrimination performance, particularly for protocols which do not manipulate nor measure convex hull, and where such protocols have facilitated certain conclusions about the ANS (e.g. that it is attainment: Halberda et al., 2008). 
Abreu-Mendoza, R. A., Soto-Alba, E. E., \& Arias-Trejo, N. (2013). Area vs. density: influence of visual variables and cardinality knowledge in early number comparison. Frontiers in Psychology, 4. https://doi.org/10.3389/fpsyg.2013.00805

400

Cappelletti, M., Didino, D., Stoianov, I., \& Zorzi, M. (2014). Number skills are maintained in healthy ageing. Cognitive Psychology, 69, 25-45. https://doi.org/10.1016/j.cogpsych.2013.11.004

Cappelletti, M., Pikkat, H., Upstill, E., Speekenbrink, M., \& Walsh, V. (2015). Learning to Integrate versus Inhibiting Information Is Modulated by Age. Journal of Neuroscience, 35(5), 22132225. https://doi.org/10.1523/JNEUROSCI.1018-14.2015

Clayton, S., \& Gilmore, C. (2014). Inhibition in dot comparison tasks. ZDM, 1-12. https://doi.org/10.1007/s11858-014-0655-2

Clayton, S., Gilmore, C., \& Inglis, M. (2015). Dot comparison stimuli are not all alike: The effect of different visual controls on ANS measurement. Acta Psychologica, 161, 177-184. https://doi.org/10.1016/j.actpsy.2015.09.007

Dakin, S. C., Tibber, M. S., Greenwood, J. A., Kingdom, F. A. A., \& Morgan, M. J. (2011). A common visual metric for approximate number and density. Proceedings of the National Academy of Sciences, 108(49), 19552-19557. https://doi.org/10.1073/pnas.1113195108

Dehaene, S. (1997). The Number Sense: How the Mind Creates Mathematics. New York: Oxford University Press.

Dehaene, S., Izard, V., \& Piazza, M. (2005). Control over non-numerical parameters in numerosity experiments. Unpublished Manuscript (available on Www.unicog.org).

DeWind, N. K., \& Brannon, E. M. (2012). Malleability of the approximate number system: effects of feedback and training. Frontiers in Human Neuroscience, 6. https://doi.org/10.3389/fnhum.2012.00068

DeWind, N. K., \& Brannon, E. M. (2016). Significant Inter-Test Reliability across Approximate Number System Assessments. Frontiers in Psychology, 7. https://doi.org/10.3389/fpsyg.2016.00310 
Dietrich, J. F., Huber, S., \& Nuerk, H.-C. (2015). Methodological aspects to be considered when measuring the approximate number system (ANS) - a research review. Cognition, 295. https://doi.org/10.3389/fpsyg.2015.00295

Folstein, M. F., Folstein, S. E., \& McHugh, P. R. (1975). “Mini-mental state”: A practical method for grading the cognitive state of patients for the clinician. Journal of Psychiatric Research, 12(3), 189-198. https://doi.org/10.1016/0022-3956(75)90026-6

Fuhs, M. W., \& McNeil, N. M. (2013). ANS acuity and mathematics ability in preschoolers from lowincome homes: contributions of inhibitory control. Developmental Science, 16(1), 136-148. https://doi.org/10.1111/desc.12013

Gallistel, C. R., \& Gelman, R. (2000). Non-verbal numerical cognition: from reals to integers. Trends in Cognitive Sciences, 4(2), 59-65. https://doi.org/10.1016/S1364-6613(99)01424-2

Gandini, D., Lemaire, P., \& Dufau, S. (2008). Older and younger adults' strategies in approximate quantification. Acta Psychologica, 129(1), 175-189. https://doi.org/10.1016/j.actpsy.2008.05.009

Gebuis, T., \& Gevers, W. (2011). Numerosities and space; indeed a cognitive illusion! A reply to de Hevia and Spelke (2009). Cognition, 121(2), 248-252.

Gebuis, T., \& Reynvoet, B. (2011). Generating nonsymbolic number stimuli. Behavior Research Methods, 43(4), 981-986. https://doi.org/10.3758/s13428-011-0097-5

Gebuis, T., \& Reynvoet, B. (2012a). Continuous visual properties explain neural responses to nonsymbolic number. Psychophysiology, 49(11), 1649-1659.

Gebuis, T., \& Reynvoet, B. (2012b). The interplay between nonsymbolic number and its continuous visual properties. Journal of Experimental Psychology: General, 141(4), 642-648. https://doi.org/10.1037/a0026218

Gebuis, T., \& Reynvoet, B. (2012c). The Role of Visual Information in Numerosity Estimation. PLoS ONE, 7(5), e37426. https://doi.org/10.1371/journal.pone.0037426 
Gebuis, T., \& van der Smagt, M. J. (2011). False Approximations of the Approximate Number System? PLoS ONE, 6(10), e25405. https://doi.org/10.1371/journal.pone.0025405

Gilmore, C., Attridge, N., Clayton, S., Cragg, L., Johnson, S., Marlow, N., ... Inglis, M. (2013). Individual Differences in Inhibitory Control, Not Non-Verbal Number Acuity, Correlate with Mathematics Achievement. PLOS ONE, 8(6). https://doi.org/10.1371/journal.pone.0067374

Gilmore, C., Cragg, L., Hogan, G., \& Inglis, M. (2016). Congruency effects in dot comparison tasks: convex hull is more important than dot area. Journal of Cognitive Psychology, 28(8), 923931. https://doi.org/10.1080/20445911.2016.1221828

Graham, R. L. (1972). An efficient algorithm for determining the convex hull of a finite planar set. Information Processing Letters, 1(4), 132-133. https://doi.org/10.1016/00200190(72)90045-2

Gray, S. A., \& Reeve, R. A. (2014). Preschoolers' Dot Enumeration Abilities Are Markers of Their Arithmetic Competence. PLoS ONE, 9(4), e94428. https://doi.org/10.1371/journal.pone.0094428

Guillaume, M., Gevers, W., \& Content, A. (2015). Assessing the Approximate Number System: no relation between numerical comparison and estimation tasks. Psychological Research, 80(2), 248-258. https://doi.org/10.1007/s00426-015-0657-x

Halberda, J., Ly, R., Wilmer, J. B., Naiman, D. Q., \& Germine, L. (2012). Number sense across the lifespan as revealed by a massive Internet-based sample. Proceedings of the National Academy of Sciences, 109(28), 11116-11120. https://doi.org/10.1073/pnas.1200196109

Halberda, J., Mazzocco, M. M. M., \& Feigenson, L. (2008). Individual differences in non-verbal number acuity correlate with maths achievement. Nature, 455(7213), 665-668. https://doi.org/10.1038/nature07246

Hasher, L., \& Zacks, R. T. (1988). Working Memory, Comprehension, and Aging: A Review and a New View. In G. H. Bower (Ed.), Psychology of Learning and Motivation (Vol. 22, pp. 193-225). 
Academic Press. Retrieved from

http://www.sciencedirect.com/science/article/pii/S0079742108600419

Hurewitz, F., Gelman, R., \& Schnitzer, B. (2006). Sometimes area counts more than number. Proceedings of the National Academy of Sciences, 103(51), 19599-19604. https://doi.org/10.1073/pnas.0609485103

Inglis, M., \& Gilmore, C. (2013). Sampling from the mental number line: How are approximate

Keller, L., \& Libertus, M. (2015). Inhibitory control may not explain the link between approximation

Izard, V., Sann, C., Spelke, E. S., \& Streri, A. (2009). Newborn infants perceive abstract numbers. Proceedings of the National Academy of Sciences, 106(25), 10382-10385. https://doi.org/10.1073/pnas.0812142106

Inglis, M., \& Gilmore, C. (2014). Indexing the approximate number system. Acta Psychologica, 145, 147-155. https://doi.org/10.1016/j.actpsy.2013.11.009

and math abilities in kindergarteners from middle class families. Frontiers in Psychology, 6. https://doi.org/10.3389/fpsyg.2015.00685

Kramer, A. F., Humphrey, D. G., Larish, J. F., \& Logan, G. D. (1994). Aging and inhibition: Beyond a unitary view of inhibitory processing in attention. Psychology and Aging, 9(4), 491-512. https://doi.org/10.1037/0882-7974.9.4.491

Leibovich, T., \& Henik, A. (2013). Magnitude processing in non-symbolic stimuli. Frontiers in Psychology, 4. https://doi.org/10.3389/fpsyg.2013.00375

Leibovich, T., \& Henik, A. (2014). Comparing performance in discrete and continuous comparison tasks. The Quarterly Journal of Experimental Psychology, 67(5), 899-917. 
Logan, G. D., \& Zbrodoff, N. J. (1979). When it helps to be misled: Facilitative effects of increasing the frequency of conflicting stimuli in a Stroop-like task. Memory \& cognition, 7(3), 166-174.

Norris, J. E., \& Castronovo, J. (2016). Dot Display Affects Approximate Number System Acuity and Relationships with Mathematical Achievement and Inhibitory Control. PLOS ONE, 11(5), e0155543. https://doi.org/10.1371/journal.pone.0155543

Norris, J. E., McGeown, W. J., Guerrini, C., \& Castronovo, J. (2015). Aging and the number sense: preserved basic non-symbolic numerical processing and enhanced basic symbolic processing. Frontiers in Psychology, 6, 999. https://doi.org/10.3389/fpsyg.2015.00999

Odic, D., Hock, H., \& Halberda, J. (2014). Hysteresis affects approximate number discrimination in young children. Journal of Experimental Psychology: General, 143(1), 255-265. https://doi.org/10.1037/a0030825

Odic, D., Libertus, M. E., Feigenson, L., \& Halberda, J. (2013). Developmental change in the acuity of approximate number and area representations. Developmental Psychology, 49(6), 11031112. https://doi.org/10.1037/a0029472

Piazza, M., \& Izard, V. (2009). How Humans Count: Numerosity and the Parietal Cortex. The Neuroscientist, 15(3), 261-273. https://doi.org/10.1177/1073858409333073

Piazza, M., Izard, V., Pinel, P., Le Bihan, D., \& Dehaene, S. (2004). Tuning Curves for Approximate Numerosity in the Human Intraparietal Sulcus. Neuron, 44(3), 547-555. https://doi.org/10.1016/j.neuron.2004.10.014

Rousselle, L., \& Noël, M.-P. (2008). The development of automatic numerosity processing in preschoolers: Evidence for numerosity-perceptual interference. Developmental Psychology, 44(2), 544-560. https://doi.org/10.1037/0012-1649.44.2.544

Spooner, J. W., Sakala, S. M., \& Baloh, R. W. (1980). Effect of aging on eye tracking. Archives of Neurology, 37(9), 575-576. https://doi.org/10.1001/archneur.1980.00500580071012

Szűcs, D., Nobes, A., Devine, A., Gabriel, F. C., \& Gebuis, T. (2013). Visual stimulus parameters seriously compromise the measurement of approximate number system acuity and 
comparative effects between adults and children. Frontiers in Psychology, 4. https://doi.org/10.3389/fpsyg.2013.00444

Warabi, T., Kase, M., \& Kato, T. (1984). Effect of aging on the accuracy of visually guided saccadic eye movement. Annals of Neurology, 16(4), 449-454. https://doi.org/10.1002/ana.410160405

West, R., \& Baylis, G. C. (1998). Effects of increased response dominance and contextual disintegration on the Stroop interference effect in older adults. Psychology and aging, 13(2), 206. 
539 Figure 1: Scatter plots showing the relationship between trials' numerosity ratios, mean accuracies

540 (\%), cumulative surface area ratios, and convex hull ratios. Ratios are plotted on logarithmic axes.

541 Ratios are calculated left array / right array so that all ratios vary below and above 1. Incongruent

542 trials are those for which the numerosity ratio is below 1 and the visual cue ratio(s) are above 1 or

543 vice-versa

544 Figure 2: Participants' accuracy on dot comparison trials showing an interaction between cumulative

545 surface area and convex hull congruency (error bars show standard error)

546 Figure 3: The impact of convex hull congruency on younger and older adults' performances (error

547 bars show standard error)

548

549

550

551

552

553

554

555

556

557

558

559 
Disclosure of interest

561 The authors report no conflicts of interest. 